\title{
Percepción de la infidelidad de adultos en el Área Metropolitana de Buenos Aires*
}

\section{Infidelity Perceptions in the Buenos Aires Metropolitan Area}

Recepción: 18 Diciembre 2015 | Aprobación: 07 Julio 2017

\author{
Ana Alejandra Fuentes Cuiñas ${ }^{\mathrm{a}}$ \\ Universidad Argentina de la Empresa, Argentina \\ ORCID: http://orcid.org/0000-0002-9349-4577 \\ Santiago Koval \\ Universidad Argentina de la Empresa, Argentina
}

a Autor de correspondencia. Correo electrónico: afuentescuinas@uade.edu.ar

Para citar este artículo: Fuentes Cuñas, A. A., \& Koval, S. (2018). Percepción de la infidelidad de adultos en el Área Metropolitana de Buenos Aires. Universitas Psychologica, 17(2), 1-9. https://doi.org/10.111 44/ Javeriana.upsy.17-2.piaa

\section{RESUMEN}

Se realizó un estudio exploratorio a 600 adultos del Área Metropolitana de Buenos Aires, vinculado con la infidelidad, tipificando la población por medio de una taxonomía de respuestas emocionales con un cuestionario online. Según estudios anteriores, y por el contrario que las mujeres, los hombres mostrarían reacciones celosas de tipo sexual y no emocional, ya que la infidelidad femenina podría conducir a la posibilidad de que tuvieran que mantener descendientes que no fueran genéticamente suyos. Las conductas se organizaron de acuerdo con la Perceptions of Dating Infidelity Scale, distinguiendo tres clases de respuestas: ambiguas, explícitas y las engañosas. Utilizando Chi cuadrado, se discuten las diferencias respecto a estas variables: la edad, el estado civil, el género, nivel educativo y creencias religiosas.

Palabras clave

infidelidad; respuestas emocionales; edad; estado civil; diferencias de género.

\begin{abstract}
An exploratory study was conducted on 600 adults from the Metropolitan Area of Buenos Aires regarded infidelity, typifying the population through a taxonomy of emotional responses with an online questionnaire. According to previous research, and opposite to women, men would show sexual and non-emotional jealousies responses, since female infidelity could lead to the possibility of having offspring that were not genetically theirs. Behaviors were organized according to the Perceptions of Dating Infidelity Scale, distinguishing three kinds of responses: ambiguous, explicit and misleading ones. Using Chi square, we discuss the differences regarding these variables: age, marital status, gender, educational level and religious practices.
\end{abstract}

Keywords

infidelity; emotional responses; age; marital status; gender differences.

De acuerdo con Hall \& Finchan (2006), la infidelidad es un acto sexual secreto, de carácter romántico o emocional, que viola el acuerdo de exclusividad en una relación. Para Platt, Nalbone, Casanova, \& Wetchler (2008), se concibe como 
aquella interacción emocional o sexual que pone en peligro la intimidad amorosa. Los vínculos sexuales extraconyugales son los actos más perjudiciales en una relación y, por ello, resultan extremadamente difíciles de tratar en las terapias de pareja (Fish, Pavkov, Wetchler, \& Bercik, 2012). Las relaciones románticas requieren de un nivel de compromiso que asegure su continuidad. La infidelidad, en este sentido, da lugar a reacciones emocionales que amenazan la estabilidad del vínculo (Cann, Mangum, \& Wells, 2001). En particular, en las relaciones amorosas de larga duración, la infidelidad conduce a riesgos en la salud (Bell, Molitor \& Flynn, 1999) y es causa y/o consecuencia del deterioro vincular (Previti \& Amato, 2004). La infidelidad, en tanto, es una de las principales causas de rupturas matrimoniales y de vínculos amorosos (Vangelisti \& Gerstenberger, 2004).

La infidelidad implica un problema significativo para muchas parejas, ya que las relaciones sexuales extraconyugales son la principal causa de disolución vincular y matrimonial (Platt, Nalbone et al., 2008; Miner \& Shackelford, 2010). En el año 2011, se registró en Buenos Aires, Argentina, una tasa del 50.5\% de divorcios, teniendo en cuenta la relación entre la cantidad de divorcios con sentencia firme y la cantidad de casamientos que se produjeron (Gobierno de la Ciudad de Buenos Aires, 2012).

Esto implica un abrupto incremento en relación con el año 2000, en el que la tasa fue del $35.8 \%$ (Gobierno de la Ciudad de Buenos Aires, 2012).

De acuerdo con los estudios de Fitch \& Ruggles (2000), la independencia laboral femenina ha dado lugar a mayores tasas de divorcios, en tanto que las mujeres ya no necesitan permanecer casadas por razones económicas. En el pasado, los matrimonios se unían por razones económicas, sociales y políticas (Coontz, 2005; Pinsof, 2002). Antes del siglo XIX, los matrimonios eran elegidos por la familia de los contrayentes, pero luego de la Revolución Industrial sus bases viraron hacia la realización personal y el amor (Coontz, 2005). Según Johnson (1999), en la actualidad, las mujeres se casan por razones personales y no necesariamente por imposiciones sociales. Esto hace que los matrimonios sean más inestables, porque, inevitablemente, en una gran parte de los casos, el amor tiende a desvanecerse con el tiempo (Kurdek, 1999). Al respecto, Campbell \& Wright (2010) sostienen que los matrimonios basados en el amor y las opciones personales son más frágiles que aquellos basados en las relaciones sociales, la economía o las motivaciones políticas. Asimismo, las relaciones entre las relaciones sexuales fuera del lazo del matrimonio o pareja y variable religiosidad no se han estudiado adecuadamente hasta el momento (Burdette, Ellison, Sherkat, \& Gore, 2007).

\section{Hombres vs. mujeres: la perspectiva evolucionista}

Según la teoría de inversión parental formulada por Trivers (1972), hombres y mujeres invierten diferentes cantidades de energía en la producción de sus genes. Por esta razón, reaccionan de diversas maneras ante mismas situaciones. Por ejemplo, mientras que los hombres dan mayor importancia a la cantidad de encuentros sexuales, las mujeres centran sus recursos en un número reducido de vínculos significativos. En este sentido, la perspectiva evolucionista sostiene que los hombres se sienten más preocupados por infidelidades de tipo sexual, mientras que las mujeres por las de tipo emocional (Buss, 1994; Buss, Larsen, Westen, \& Semmelroth, 1992). Asimismo, los hombres tienden a describir la infidelidad de un modo más sexual que emocional, mientras que las mujeres lo hacen de modo contrario (Glass \& Wright, 1985). De acuerdo a García Leiva, Gómez Jacinto, y Canto Ortiz (2001), las diferencias entre hombres y mujeres ante el tipo de infidelidad, sexual o emocional, son estudiadas principalmente por dos grandes teorías: la sociocultural y evolucionista.

Según Pinker (1997), uno de los módulos innatos específicos de la naturaleza dimórfica de la mente se encarga de resolver situaciones asociadas a los celos y permite reaccionar en forma emocional, cognitiva y conductual ante distintas clases de infidelidad. Los celos son 
mecanismos destinados a la conservación de la pareja y suelen ser, en tanto, una de las principales causas de disolución de vínculos amorosos (Buss \& Shackerford, 1997). La mujer tiene siempre la certeza de que el hijo que espera es suyo, mientras que para el hombre esta seguridad no existe. En este sentido, puede explicarse la reacción emocional en los celos de las mujeres, en tanto que la infidelidad del hombre podría implicar un desvío de los recursos para la manutención de hijos que ha tenido con otras parejas. Por el contrario, los hombres mostrarían reacciones celosas de tipo sexual y no emocional, ya que la infidelidad femenina podría conducir a la posibilidad de que tuvieran que mantener descendientes que no fueran genéticamente suyos.

La perspectiva evolucionista, de corte biológico, ha sido complementada por la "hipótesis del doble shock", de corte sociológico, formulada por Treger \& Sprecher (2011). Esta hipótesis sostiene que hombres y mujeres perciben los modos de socialización de manera diferenciada. Las mujeres consideran que los hombres pueden mantener relaciones sexuales sin establecer vínculos emocionales; no obstante, conciben que si el hombre se vincula emocionalmente con una mujer, el vínculo será necesariamente más completo y duradero. Por este motivo, las mujeres se sienten más amenazadas por una infidelidad emocional y no por un engaño de tipo sexual. Por el contrario, los hombres suelen pensar que las mujeres pueden vincularse emocionalmente con un hombre sin que medie en ello una relación sexual y que mantendrán, además, un vínculo sexual solamente con aquellos hombres con los que hayan establecido un enlace emocional. Por ello, los hombres se sienten más amenazados por una infidelidad sexual y no por un engaño de tipo emocional. Los hombres son menos propensos a perdonar y a olvidar una infidelidad sexual y, por tanto, tienen mayor predisposición que las mujeres a disolver una relación sentimental por este motivo.

La jerarquización diferenciada entre lo sexual y lo intelectual se puede comprender a partir de la “teoría triárquica del amor” de Sternberg (1998), quien postula la existencia de tres componentes centrales en las relaciones adultas: la intimidad, la pasión y el compromiso personal.

Sin embargo, más allá de las diferencias biológicas y sociológicas entre géneros, los resultados de las investigaciones de Canto Ortiz, García Leiva, y Jacinto (2009) indican que las mujeres manifiestan, en general, una reacción más intensa que los hombres ante cualquier tipo de infidelidad amorosa. $\mathrm{Al}$ respecto, en una investigación realizada por Whitty (2003), en la que se puntuaron comportamientos de acuerdo con una escala gradual (desde "no es considerado infidelidad" a "extrema infidelidad"), las mujeres resultaron ser mucho más severas que los hombres. Asimismo, en un estudio llevado a cabo por la Universidad de Granada, se demostró que los hombres se encuentran significativamente más preocupados por una infidelidad de tipo sexual mientras que las mujeres lo hacen por una infidelidad de tipo emocional (Fernández, VeraVillarroel, Sierra, \& Zubeidat, 2007).

Una posible explicación que puede dar cuenta de esta diferencia de apreciación entre hombres y mujeres se vincula con la denominada perspectiva sociohistórica. De acuerdo a DeSteno \& Salovey (1996), las personas determinan que un tipo de infidelidad (sexual o emocional) es la más angustiante, basadas en el diferencial percibido de esta misma diferencia en su pareja. Los autores anteriormente mencionados también defienden y concuerdan con la postura de Harris \& Christenfeld (1996), quienes a su vez también defienden la importancia de medir las diferencias sexuales.

\section{Objetivos e hipótesis de estudio}

El objetivo consistió en diseñar e implementar una investigación por encuestas que permitiera ensayar un diagnóstico acerca de la percepción de las distintas conductas asociadas al constructo infidelidad en el Área Metropolitana de Buenos Aires (AMBA). La finalidad fue generar un aporte fresco, apartándonos de las clásicas taxonomías referidas a explicaciones de 
infidelidad relacionadas únicamente al género, por considerarlo a priori un enfoque trillado.

Teniendo en cuenta el malestar y la posible disolución de la pareja que conlleva la infidelidad, el proyecto se propuso ser un primer acercamiento a la comprensión del modo en que la población de estudio clasifica aspectos centrales del imaginario vinculado con la reacción emocional asociada a la infidelidad en el AMBA. Así, el objetivo fue el de ubicar en la distinción de la taxonomía descripta por Wilson, Mattingly, Clark, Weidler, \& Bequette (2011) respecto a las distintas variables de clasificación de la población de AMBA. Puntualmente, el objetivo fue el de analizar las siguientes variables: la edad, el estado civil, el género, nivel educativo, estado no civil y las prácticas de propia infidelidad. Respecto a las hipótesis, y de acuerdo a la bibliografía, se hipotetiza que las personas serán más severas a la hora de considerar las conductas relacionadas con la infidelidad cuanto mayor cantidad de años tengan, si son casados, si son de género femenino, si poseen un alto nivel educativo y si son creyentes.

\section{Método}

Se presenta un estudio exploratorio orientado a comprender el modo en que la población del AMBA clasifica aspectos centrales del imaginario vinculado con la infidelidad en la pareja amorosa. Metodológicamente, el abordaje se concentró en clasificar a la población por medio de una taxonomía de conductas y de respuestas emocionales ante diversos tipos de infidelidad amorosa. El trabajo de campo se abordó por medio de un muestreo aleatorio estratificado de la población del AMBA y se condujo a través de encuestas electrónicas autoadministradas. El universo de estudio está compuesto por hombres y mujeres de edad mayor o igual a los 18 años, residentes en el AMBA (10.042.619). El marco muestral se compone por los habitantes del universo con acceso a una computadora, es decir, un total de 7.176.048 de habitantes, según datos del Censo 2010 de Argentina. Se operó un abordaje muestral estratificado por género, edad, nivel educativo y estado civil.

\section{Participantes}

La muestra se compone de 600 sujetos del AMBA. La mayoría reside en la Ciudad Autónoma de Buenos Aires (56\%), es soltera (52 $\%)$ o casada (39\%), y tiene un nivel educativo secundario (40\%) o universitario (38\%). El 72 $\%$ es menor de 35 años y el $43 \%$, menor de 25 ; el $50 \%$ es de género masculino y el $50 \%$, del género femenino. El $75 \%$ se encuentra una relación amorosa, el $47 \%$ convive con su pareja y el $28 \%$ vive en una casa distinta; en promedio, estas relaciones amorosas llevan entre 3 y 5 años de duración. En cuanto a las creencias religiosas, el $70 \%$ se define como creyente, de los cuales el $25 \%$ dice ser practicante. El $60 \%$ afirma no haber sido nunca infiel y más del $62 \%$ jamás haberse enterado de que hayan sido engañados.

\section{Instrumento}

La percepción de la infidelidad puede medirse mediante la apreciación de conductas asociadas al imaginario de lo infiel. En este estudio, las conductas se organizaron de acuerdo con la Perceptions of Dating Infidelity Scale (Wilson et al., 2011), que distingue tres clases genéricas de conducta: por un lado, las ambiguas: conductas que no se asocian de forma clara con la infidelidad, pero en las que puede existir una posibilidad de traición; las explícitas: conductas típicamente asociadas a la infidelidad; y las engañosas: conductas que se llevan a cabo sin que exista conciencia por parte del/a compañero/a amoroso/a y que pueden implicar comportamientos infieles. A continuación se presentan los resultados de las tres subescalas: Conductas ambiguas (por ejemplo comer o tomar algo, bailar con alguien que no sea la pareja, etc.; $\alpha=0.72$ ); conductas explicitas (por ejemplo citas, relaciones sexuales, etc.; $\alpha=0.73$ ) y por ultimo conductas engañosas (por ejemplo citas, esconder información de la pareja, etc.; $\alpha=0.85$ ). 
El listado de conductas utilizadas para la presente investigación se generó a partir de la revisión de la literatura y de las investigaciones realizadas previamente, principalmente en Estados Unidos. Se reconocieron las siguientes conductas organizadas de acuerdo con la escala PDIS: ambiguas (hacer una salida romántica, abrazarse emotivamente, bailar románticamente, intercambiar miradas cómplices, dar o recibir regalos interesadamente, vestirse deliberadamente para una salida); explícitas (tener sexo, dar o recibir sexo oral, pagar por sexo, besarse apasionadamente); engañosas (mirar pornografía, tener sexo virtual, fantasear, mentir, ocultar información, hablar por teléfono a escondidas, chatear sobre cuestiones íntimas, eliminar mensajes de texto en el celular, eliminar el historial de navegación en la computadora). Se incluyeron, también, preguntas cuya finalidad fue la de averiguar con qué persona específica les molestaría más ser engañados.

\section{Diseño}

La investigación tuvo un diseño no experimental de tipo descriptivo-correlacional. La muestra fue tomada por conveniencia a través del método de bola de nieve, pidiendo que los contactados que lo reenvíen a otros conocidos, a fin de dar con la mayor cantidad y variedad posible de personas que respondieran.

\section{Procedimiento}

En primer lugar, se realizó una prueba piloto con 20 respondedores, donde se observó que no había mayores inconvenientes. Luego se procedió a tomar la muestra, dividiendo el en tres conjuntos de preguntas. El primero se destinó a especificar dimensiones sociodemográficas (género, edad, nivel educativo, estado civil, etc.); el segundo, a solicitar una clasificación, según niveles de intensidad, de conductas asociadas a la infidelidad; el tercero, a indagar en determinadas respuestas emocionales ante situaciones hipotéticas específicas. Desde un punto de vista general, la herramienta se estructuró con arreglo a la detección de correlaciones entre características sociodemográfica y la clasificación subjetiva de conductas y respuestas emocionales en situación de engaño. La hipótesis de base es que diversos perfiles poseen escalas valorativas particulares y que perciben, por tanto, el imaginario de lo infiel de modos diferenciados. El objetivo, dado este esquema, radicó en identificar las variables de perfil que generan mayores diferencias de apreciación.

En el segundo segmento de la ficha, se listaron 19 conductas asociadas a la infidelidad. Con el objeto de medir grados de intensidad en su asociación al concepto de lo infiel, se aplicó una escala gradual destinada a dimensionar la intensidad asociativa. El sujeto entrevistado debió valorar cada conducta según niveles de intensidad a partir de una escala ordinal de cinco valores: del 1 ("baja intensidad") al 5 ("alta intensidad"). A mayor nivel de intensidad, mayor fuerza en la relación que se establece entre conducta y concepto. En la etapa de análisis, estos ítems se clasificaron de acuerdo con la Perceptions of Dating Infidelity Scale. El sentido del análisis, en este aspecto, radicó, por un lado, en identificar concurrencias estadísticamente significativas entre variables sociodemográficas y las tres clases de conductas y, por otro, en operar un análisis factorial exploratorio que permita corroborar la viabilidad de aplicar el modelo norteamericano a la población del AMBA.

En el tercer segmento de la ficha, se midieron respuestas emocionales ante situaciones hipotéticas de infidelidad a partir de la siguiente pregunta: "icon quién le molestaría más que le fueran infiel?" Se indagó en respuestas vinculadas con aquellas reacciones asociadas al género. La pregunta requirió la indicación excluyente del grupo sexual que produciría mayor molestia en situación de infidelidad, esto es: "con alguien del género opuesto al de mi pareja", "con alguien del mismo género de mi pareja" o, en caso de indiferencia, "es lo mismo". El propósito del análisis consistió aquí en reconocer concurrencias entre variables sociodemográficas y respuestas emocionales. 


\section{Consentimiento informado}

Dado que la encuesta fue autoadministrada, se ha incluido al comienzo de la misma un párrafo explicando los objetivos de la investigación y solicitando la aceptación. En caso de las personas que han marcado la opción de no aceptación, se dio por concluida la encuesta.

\section{Análisis de resultados}

Los resultados fueron analizados a través del programa estadístico SPSS, versión 20.

\section{Resultados}

\section{Figura 1}

Taxonomía de conductas de acuerdo con la Perceptions of Dating Infidelity Scale. Nota. Los valores corresponden a las puntuaciones medias

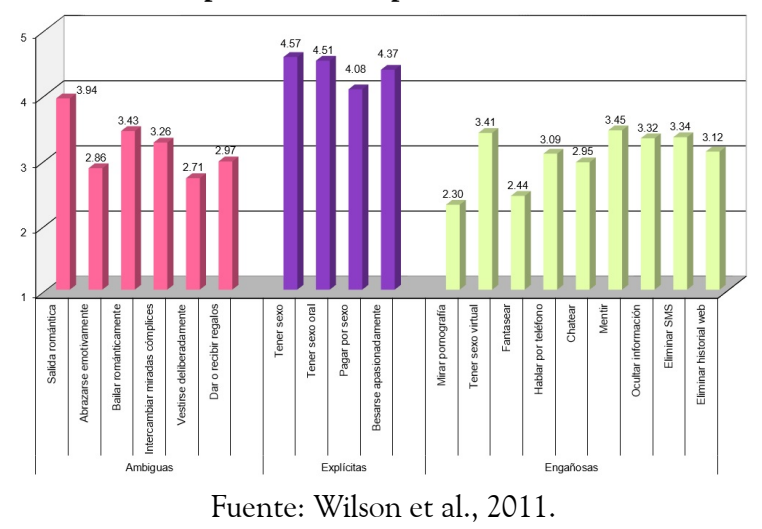

Taxonomía de reacciones emocionales

En la Figura 1 se presenta la clasificación de las emociones en función a los distintos ítems de la escala. En una escala se midieron cada una de las variables con una escala Likert, pudiéndose puntuar las mismas del 1 al 5, siendo 1 "baja intensidad" y 5 "alta intensidad". Así, se encontraron estas medidas estadísticas: conductas ambiguas $(X=3.19 ; D E=0.75)$; las explicitas $(X=4.37 ; D S=0.95)$ y las engañosas $(X=3.04 ; D E=0.79)$.
En los cruces por pruebas de Chi Cuadrado, según se detalla a continuación, se detectaron relaciones significativas con cinco variables sociodemográficas respecto a taxonomía descripta: la edad, el estado civil, el género, nivel educativo y creencias religiosas.

Respecto de la edad, se encontraron relaciones significativas respecto a las conductas engañosas $\left(X^{2}(240)=336.545 ; p=0\right)$; las conductas ambiguas $\left(X^{2}(240)=230.754 ; p=0.001\right)$, pero no se encontraron dichas diferencias en el caso de las conductas explicitas $\left(X^{2}(240)=120.125\right.$; $p=0.083$ ).

Respecto al estado civil, no se encontraron relaciones significativas respecto a ninguna de las taxonomías: conductas engañosas $\left(X^{2}(96)=\right.$ $96.455 ; p=0.468)$, ambiguas $\left(X^{2}(66)=74.126\right.$; $p=0.23)$ y explicitas $\left(X^{2}(40)=51,021 ; p=\right.$ $0.114)$.

Respecto al género, se encontraron relaciones significativas solamente respecto a las conductas engañosas $\left(X^{2}(48)=68.002 ; p=0.03\right)$, pero no con las ambiguas $\left(X^{2}(33)=40.414 ; p=0.175\right)$ ni las explicitas $\left(X^{2}(20)=20.826 ; p=0.407\right)$.

Respecto al nivel educativo, se encontraron relaciones significativas respecto a las conductas engañosas $\left(X^{2}(144)=196.172 ; p=0.003\right)$; las conductas ambiguas $\left(X^{2}(99)=117.550 ; p=\right.$ 0.098), pero no se encontraron dichas diferencias en el caso de las conductas explicitas $\left(X^{2}(60)=\right.$ 74.044; $p=0.105$ ).

Por último, respecto al nivel de compromiso con las prácticas religiosas, estos fueron los resultados encontrados: conductas engañosas $\left(X^{2}(96)=100.926 ; p=0.345\right)$, ambiguas $\left(X^{2}\right.$ $(66)=72.666 ; p=0.268)$ y explicitas $\left(X^{2}(40)\right.$ $=51.769 ; p=0.101)$. Como puede observarse, en ninguna de las tres taxonomías se hallaron diferencias significativas en esta variable.

Aplicando ANOVA, se encontró una diferencia estadísticamente significativa en la taxonomía Conducta engañosas descriptas con la variable género $\left(F(2,590)=18.007 ; p<0 ; \eta^{2}\right.$ $=0.989$ ). Se encontraron diferencias entre las mujeres y los hombres, en favor del primer grupo. 


\section{Discusión}

En términos generales, y tal como se esperaba en función del estudio realizado por Wilson et al. (2011), las conductas explícitas (conductas típicamente asociadas a la infidelidad) reciben una valoración más severa que el resto de las conductas. Asignar una valoración más severa significa, en este contexto, que las conductas se asocian de forma más intensa al concepto de la infidelidad. En segundo lugar se ubicaron las conductas ambiguas (conductas que no se asocian de forma clara con la infidelidad, pero en las que puede existir una posibilidad de traición) y en tercer término, las conductas engañosas (conductas que se llevan a cabo sin que exista conciencia por parte del compañero amoroso y que pueden implicar comportamientos infieles). Aunque es prudente decir que no se observan grandes diferencias entre estas dos últimas variables mencionadas. Por otro lado, es poco lo que se sabe en la bibliografía respecto a los cruces realizados, por eso se plantean posibles respuestas, con la idea que puedan ser abordados en próximos estudios.

Respecto a la edad se encontraron relaciones significativas respecto a las conductas engañosas y ambiguas, pero no en el caso de las conductas explicitas. Quizás esto se deba a que las conductas mencionadas no dejan duda acerca de la infidelidad.

Resulta extraño no encontrar relaciones significativas según el estado civil. Podría deberse a las tasas decrecientes de casamientos y las altas tasas de divorcios (Gobierno de la Ciudad de Buenos Aires, 2012).

En lo que refiere al nivel educativo, se encontraron diferencias entre las conductas engañosas y ambiguas, pero no en las explicitas. Podría pensarse que esto se debe a que las personas con mayor nivel educativo tienen la posibilidad de utilizar y comprender de otra forma las distintas vías y formas de poder llevar cabo las conductas relacionadas con la infidelidad.

El género fue tal vez la mayor sorpresa, habida cuenta que mucho se ha dicho acerca de las diferencias relativas a esta variable en materia de infidelidad. Es difícil inferir una conclusión al respecto, no obstante, puede reflexionarse que tal vez estos resultados se encuentran vinculados con la libertad económica actual que gozan muchas mujeres en la actualidad, que se asemeja cada día más a los hombres. Tal como comentan Fitch \& Ruggles (2000), la independencia laboral femenina ha dado lugar a mayores tasas de divorcios, teniendo en cuenta que muchas mujeres no se encuentran obligadas a mantener una relación con un hombre a fin de saciar sus necesidades materiales, a diferencia de otras épocas, donde las mujeres se unían en matrimonio meramente por razones económicas, sociales y políticas (Coontz, 2005; Pinsof, 2002).

Para finalizar, en lo que refiere a cuestiones relacionadas con la religiosidad, tampoco se encontró la hipótesis relacionada a que las personas más religiosas se mostrarían más estrictas a la hora de considerar las conductas vinculadas con la infidelidad de aquellas que no se reportan como practicantes de alguna religión. Pero, tal como se anticipó citando el artículo de Burdette et al. (2007), no debiera sorprender esta cuestión, al tenerse en cuenta que es una temática poco estudiada.

En lo que refiere a la mirada general de la infidelidad, solo hemos encontrado asociaciones entre las mujeres y los hombres en lo que refiere a las conductas engañosas. Esto está en línea, al menos en parte, con lo expresado por Glass y Wright (1985), ya que habla que los hombres tienden a describir la infidelidad de un modo más sexual que emocional, mientras que las mujeres lo hacen de modo contrario. No obstante, el que no se hayan encontrado diferencias significativas en las demás taxonomías quizás confirme lo expuesto por Fitch \& Ruggles (2000) acerca de la independencia laboral femenina.

Tal como sostienen Fish et al. (2012), las relaciones sexuales por fuera de la pareja son los actos más perjudiciales para el bienestar de la pareja. Así, también en Buenos Aires, la infidelidad implica un problema significativo para muchas parejas, ya que este acto representa la principal causa de disolución vincular (Platt et al., 2008; Miner \& Shackelford, 2010). Por todo lo expuesto hasta el momento, los autores consideran de utilidad tener un conocimiento 
más cabal de los aspectos relacionados con el fenómeno de infidelidad.

\section{Limitaciones}

Teniendo en cuenta que este estudio ha sido exploratorio de la importancia a nivel emocional que las conductas de infidelidad generan en las parejas, parecería interesante contar con los resultados a nivel del país, y no solamente en Buenos Aires. Asimismo, y como extensión de lo anteriormente dicho, podría resultar relevante poder comparar los resultados entre distintos tipos de poblaciones, especialmente las posibles diferencias en el imaginario social de las zonas rurales y citadinas. Por otro lado, también sería interesante analizar diferencias entre distintos países de Latinoamérica, e incluso entre otras regiones, sobre todo, tomando como incide el hecho que en ámbitos latinoamericanos el contacto físico o afectivo no es considerado necesariamente sexual o indicador de infidelidad. Para tal fin, sería de alto valor el hecho de validar la escala al español, especialmente porque no es posible entender con claridad las diferencias entre la taxonomía engañosa y ambigua. Por otro lado, sería conveniente contar con investigaciones que permitan analizar con mayor claridad qué variables inciden en la percepción de infidelidad.

\section{Referencias}

Bell, R. A., Molitor, F., \& Flynn, N. M. (1999). Fear of AIDS: Assessment and implications for promoting safer sex. AIDS and Behavior, 3(2), 135-147.

Burdette, A. M., Ellison, C. G., Sherkat, D. E., \& Gore, K. A. (2007). Are there religious variations in marital infidelity? Journal of Family Issues, 28(12), 1553-1581.

Buss, D. (1994). The evolution of desire: Strategies of human mating. Nueva York: Basic Books.

Buss, D. M., Larsen, R. J., Westen, D., \& Semmelroth, J. (1992). Sex differences in jealousy: Evolution, physiology, and psychology. Psychological Science, 3(4), 251-255.

Buss, D., \& Shackelford, T. K. (1997). From vigilance to violence: Mate retention tactics in married couples. Journal of Personality and Social Psychology, 72(2), 346-361.

Canto Ortiz, J. M., García Leiva, P., \& Jacinto, L. (2009). Celos y emociones: Factores de la relación de pareja en la reacción ante la infidelidad. Athenea Digital (Revista De Pensamiento E Investigación Social), 15, 39-55.

Campbell, K., \& Wright, D. W. (2010). Marriage Today: Exploring the Incongruence Between Americans' Beliefs and Practices. Journal Of Comparative Family Studies, 41 (3), 329-345.

Cann, A., Mangum, J. L., \& Wells, M. (2001). Distress in Response to Relationship Infidelity: The Roles of Gender and Attitudes about Relationships. Journal Of Sex Research, 38(3), 185-190.

Coontz, S. (2005). Marriage, a History: How love conquered marriage. Nueva York: Viking.

DeSteno, D. A., \& Salovey, P. (1996). Jealousy and the characteristics of one's rival: A self-evaluation maintenance perspective. Personality and Social Psychology Bulletin, 22 (9), 920-932.

Fernández, A., Vera-Villarroel, P., Sierra, J., \& Zubeidat, I. (2007). Distress in Response to Emotional and Sexual Infidelity: Evidence of Evolved Gender Differences in Spanish Students. Journal Of Psychology, 141(1), 17-24.

Fish, J. N., Pavkov, T. W., Wetchler, J. L., \& Bercik, J. (2012). Characteristics of Those Who Participate in Infidelity: The Role of Adult Attachment and Differentiation in Extradyadic Experiences. American Journal Of Family Therapy, 40(3), 214-229. https:// doi.org/10.1080/01926187.2011.601192.

Fitch, C. A., \& Ruggles, S. (2000), Historical trends in marriage formation: The United States 1850-1990. En L. J. Waite (Ed.), The ties that bind: Perspectives on marriage 
and cohabitation (pp. 59-88). Nueva York: Aldine de Gruyter.

García Leiva, P., Gómez Jacinto, L., \& Canto Ortiz, J. M. (2001). Reacción de celos ante una infidelidad: diferencias entre hombres y mujeres y características del rival. Psicothema, 13(4), 611-616.

Glass, S., \& Wright, T. (1985). Sex differences in type of extramarital involvement and marital dissatisfaction. Sex Roles, 12 (9-10), 1101-1120.

Gobierno de la Ciudad de Buenos Aires. (2012, Junio). Los divorcios en la ciudad de Buenos Aires. Informe de Resultados 508. Recuperado de: http://estatico.buenosaires.gov.ar/areas /hacienda/sis_estadistico/ir_2012_508.pdf Consultado 27/07/12.

Hall, J. H., \& Fincham, F. D. (2006). Relationship dissolution following infidelity: the roles of attributions and forgiveness. Journal of Social Eु Clinical Psychology, 25(5), 508-522.

Harris, C. R., \& Christenfeld, N. (1996). Gender, jealousy, and reason. Psychological Science, 7(6), 364-366.

Johnson, M. P. (1999). Personal, moral, and structural commitment to relationships: Experiences of choice and constraint. En J. M. Adams, \& W. H. Jones (Eds.), Handbook on interpersonal commitment and relationship stability (pp. 73-87). Nueva York: Kluwer Academic.

Kurdek, L. A. (1999). The nature and predictors of the trajectory of change in marital, quality for husbands and wives over the first 10 years of marriage. Developmental Psychology, 35(5), 1283-1296.

Miner, E. J., \& Shackelford, T. K. (2010). Mate attraction, retention and expulsion. Psicothema, 22(1), 9-14.

Pinsof, W. M. (2002). The death of "till death do us part": The transformation of pairbonding in the 20th century. Family Process, $41(2), 135-157$.

Pinker, S. (1997). How the mind works. Nueva York: Norton.

Platt, R. L., Nalbone, D. P., Casanova, G. M., \& Wetchler, J. L. (2008). Parental Conflict and Infidelity as Predictors of Adult Children's Attachment Style and Infidelity. American Journal of Family Therapy, 36(2), 149-161. https://doi.org/10.1080/01926180 701236258

Previti, D., \& Amato, P. (2004). Is Infidelity a Cause or a Consequence of Poor Marital Quality? Journal of Social and Personal Relationships, 21 (2), 217-230.

Sternberg, R. (1988). The triangle of love. Nueva York: Basic Books.

Treger, S., \& Sprecher, S. (2011). The Influences of Sociosexuality and Attachment Style on Reactions to Emotional Versus Sexual Infidelity. Journal of Sex Research, 48(5), 413-422. https://doi.org/10.1080/00224499 .2010 .516845

Trivers, R. L. (1972). Parental investment and sexual selection. En B. Campbell (Ed.), Sexual selection and the descent of man (pp. 136-179). Chicago: Adline.

Vangelisti, A. L., \& Gerstenberger, M. (2004). Communication and marital infidelity. En J. Duncombe, K. Harrison, G. Allan, \& D. Marsden (Eds.), The state of affairs: Explorations in infidelity and commitment (pp. 59-78). Mahwah: Erlbaum.

Wilson, K., Mattingly, B. A., Clark, E. M., Weidler, D. J., \& Bequette, A. W. (2011). The gray area: Exploring attitudes toward infidelity and the development of the Perceptions of Dating Infidelity Scale. The Journal of social psychology, 151(1), 63-86.

Whitty, M. T. (2003). Pushing the wrong buttons: Men's and women's attitudes toward online and offline infidelity. CyberPsychology and Behavior, 6(6), 569-579

\section{Notas}

* Artículo de investigación. 